\title{
Chiral anomaly, Berry phase, and chiral kinetic theory from worldlines in quantum field theory
}

\author{
Niklas Mueller ${ }^{1,2, *}$ and Raju Venugopalan ${ }^{2, \dagger}$ \\ ${ }^{1}$ Institut für Theoretische Physik, Universität Heidelberg, Philosophenweg 16, 69120 Heidelberg, Germany \\ ${ }^{2}$ Physics Department, Brookhaven National Laboratory, Building 510A, Upton, New York 11973, USA
}

(Received 19 February 2017; published 21 March 2018)

\begin{abstract}
In previous work, we outlined a worldline framework that can be used for systematic computations of the chiral magnetic effect (CME) in ultrarelativistic heavy-ion collisions. Towards this end, we first expressed the real part of the fermion determinant in the QCD effective action as a supersymmetric worldline action of spinning, colored, Grassmanian point particles in background gauge fields, with equations of motion that are covariant generalizations of the Bargmann-Michel-Telegdi and Wong equations. The chiral anomaly, in contrast, arises from the phase of the fermion determinant. Remarkably, the latter too can be expressed as a point particle worldline path integral, which can be employed to derive the anomalous axial vector current. We will show here how Berry's phase can be obtained in a consistent nonrelativistic adiabatic limit of the real part of the fermion determinant. Our work provides a general first principles demonstration that the topology of Berry's phase is distinct from that of the chiral anomaly confirming prior arguments by Fujikawa in specific contexts. This suggests that chiral kinetic treatments of the CME in heavy-ion collisions that include Berry's phase alone are incomplete. We outline the elements of a worldline covariant relativistic chiral kinetic theory that captures the physics of how the chiral current is modified by manybody scattering and topological fluctuations.
\end{abstract}

DOI: 10.1103/PhysRevD.97.051901

\section{INTRODUCTION}

The possibility that topological sphaleron transitions can be identified in heavy-ion collision experiments has aroused great interest. Besides the information they provide about the nonperturbative real time dynamics of the QCD vacuum, sphaleron transitions are conjectured to play a role in electroweak baryogenesis [1,2]. A striking manifestation of the role of topology is the chiral magnetic effect (CME) where, as a consequence of the chiral anomaly, an induced current is generated in the direction of the external magnetic field $[3,4]$. Whether such an effect is seen in heavy-ion collisions is still unclear and is a focus of experimental research in the field $[5,6]$. We note that the CME has been observed in condensed matter systems [7].

For the CME to be large enough to be observed, it must be generated at the earliest times in the heavy-ion collision where the magnetic fields are very large initially, before dying off rapidly [8,9]. First principles weak coupling computations of sphaleron transitions [10] in the produced

\footnotetext{
*nmueller@bnl.gov

raju@bnl.gov
}

Published by the American Physical Society under the terms of the Creative Commons Attribution 4.0 International license. Further distribution of this work must maintain attribution to the author(s) and the published article's title, journal citation, and DOI. Funded by SCOAP ${ }^{3}$. nonequilibrium Glasma matter indicate that the sphaleron transition rate is significantly larger. ${ }^{1}$ than the corresponding equilibrium rate [11]. Because the occupancies of gluons in the Glasma are large, classical-statistical simulations can be employed [12] to compute $a b$ initio, in a sphaleron background, and in the presence of external magnetic fields $[13,14]$, the development of a chiral magnetic, and accompanying "chiral separation," wave $[15,16]$.

Detailed thermalization scenarios however suggest that thermalization occurs at parametrically later times $[17,18]$. Because the gluon occupancy is of order unity or lower in this regime, kinetic theory provides the appropriate description of quark-gluon dynamics. The classical-statistical simulations, that are valid for the early time description of the CME, must therefore be matched to this framework. Likewise, at even later times, when the quark-gluon matter is strongly coupled, the kinetic description of chiral currents must be matched to anomalous hydrodynamics [19-22].

There has been a considerable amount of recent work on chiral kinetic theory, and of the role therein of the well known Berry phase [23] and of the chiral anomaly [24-42]. We will discuss here a first principles worldline approach developed by us [43] toward deriving a covariant kinetic theory in QCD (or even QED). In this worldline approach,

\footnotetext{
${ }^{1}$ The computations are for over-occupied SU(2) gauge fields in a fixed box. More realistic geometries and $N_{c}=3$ will not modify this qualitative conclusion.
} 
it is manifest that the topology of Berry's phase is distinct from that of the chiral anomaly. The two are often conflated, a point that has been made previously in the context of specific condensed matter systems by Fujikawa and collaborators [44-46]. Our work leaves no room for ambiguity. We will show that Berry's phase arises from the real part of the worldline effective action in a particular nonrelativistic adiabatic limit. In contrast, the chiral anomaly is obtained from the imaginary part of the worldline effective action. The proper identification of the two effects is not academic but is important for the treatment of topological fluctuations experienced by a chiral current traversing the hot and dense fireball created in heavy-ion collisions.

We further outline key elements in the worldline formalism necessary for a first principles derivation of a relativistically covariant kinetic theory. One such element is provided by the Euler-Lagrange equations of motion derived from the worldline action ${ }^{2}$ for the spinning and colored (in the QCD case) Grassmanian fields [47-56]. Variation of the worldline action for spinning particles yields the well know Bargmann-Michel-Telegdi equations [47-51,57]. Further, by embedding the saddle point describing worldline trajectories in the SchwingerKeldysh real time formulation [58,59], one obtains classical many-body phase space of fermions. In this generalized formulation of phase space, the internal spin degrees of freedom are anticommuting coordinates [55]. Employing a standard fluctuation analysis in the Schwinger-Keldysh framework, we are able make contact with the Boltzmann transport equation, where we identify the collision terms. While the dynamics of spinning particles can be formulated in terms of an extended phase space, the source of the anomaly however is found in the structure of worldline quantization itself. A self-consistent derivation of the detailed structure of the many-body kinetic theory for spinning particles, and of the topological fluctuations induced by the QCD axial anomaly, is out of the scope of this work and will be reported on in the near future [60].

\section{REVIEW OF THE WORLDLINE FORMALISM IN QED}

We will begin by briefly reviewing aspects of the worldline formalism necessary for the construction of a chiral kinetic theory. These were discussed by us at greater length in [43]. For simplicity, we will consider QED; the extension to QCD is straightforward. The real part of the fermion determinant can be expressed exactly in terms of the supersymmetric quantum mechanical path integral for point particle worldlines [58,61-67], where the internal

\footnotetext{
${ }^{2}$ Prior work in this context was motivated from either symmetry considerations [47-51] or Schwinger's proper time representation of the propagator [52-54].
}

spin and color degrees (in QCD) of freedom are expressed in terms of Grassmann variables [55,56].

We begin with the Euclidean action for massless fermion fields in the background of a vector $(A)$ and an auxiliary Abelian axial-vector $(B)$ field $^{3}$

$$
S_{F}[A, B]=\int d^{4} x \bar{\psi}\left(i \not+\not A+\gamma_{5} \not \subset\right) \psi,
$$

and allow the fermion fields to transform under internal (gauge) symmetry. Performing the path integral over the fermion fields, one obtains the effective action

$$
-W[A, B]=\log \operatorname{det}(\theta) \quad \theta=i \partial+\not A+\gamma_{5} \not B .
$$

The fermion determinant has a relative phase that is well known to be related to the physics of the chiral anomaly [68-71]. We will here adapt a trick due to D'Hoker and Gagné $[72,73]$, which provides an alternative derivation to those often discussed in the literature. One begins by formally splitting Eq. (2) into real and imaginary parts,

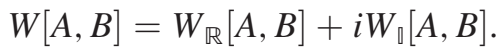

The real part of the effective action can expressed as

$$
W_{\mathbb{R}}=-\frac{1}{2} \log \operatorname{det}\left(\theta^{\dagger} \theta\right) .
$$

As shown in $[72,73]$, this can be rewritten as

$$
W_{\mathbb{R}}=-\frac{1}{8} \log \operatorname{det}\left(\tilde{\Sigma}^{2}\right)=-\frac{1}{8} \operatorname{Tr} \log \left(\tilde{\Sigma}^{2}\right) .
$$

Here $\tilde{\Sigma}^{2}$ is a sixteen dimensional matrix given by

$$
\tilde{\Sigma}^{2}=(p-\mathcal{A})^{2} \rrbracket_{8}+\frac{i}{2} \Gamma_{\mu} \Gamma_{\nu} F_{\mu \nu}[\mathcal{A}],
$$

with $\square_{8}$ the 8-dimensional identity matrix and

$$
\begin{aligned}
\Gamma_{\mu}=\left(\begin{array}{cc}
0 & \gamma_{\mu} \\
\gamma_{\mu} & 0
\end{array}\right), \quad \Gamma_{5}=\left(\begin{array}{cc}
0 & \gamma_{5} \\
\gamma_{5} & 0
\end{array}\right), \\
\Gamma_{6}=\left(\begin{array}{cc}
0 & i \rrbracket_{4} \\
-i \rrbracket_{4} & 0
\end{array}\right),
\end{aligned}
$$

are $8 \times 8$ dimensional gamma matrices. We further define a $\Gamma_{7}$ matrix anticommuting with all other elements of the algebra,

$$
\Gamma_{7}=-i \prod_{A=1}^{6} \Gamma_{A}=\left(\begin{array}{cc}
\mathbb{a}_{4} & 0 \\
0 & -\mathbb{\square}_{4}
\end{array}\right) .
$$

The gauge fields in Eq. (5) that appear explicitly and in the field-strength tensor $F_{\mu \nu}[\mathcal{A}]$ can be split into left-right chiral structures with the $2 \times 2$ dimensional matrix form, ${ }^{4}$

\footnotetext{
${ }^{3}$ Couplings are absorbed into the definitions of the fields.

${ }^{4}$ The $8 \times 8$ Gamma matrices in $\tilde{\Sigma}^{2}$ should be understood as multiplying the diagonal terms of the $2 \times 2$ matrices formed by the gauge fields.
} 


$$
\mathcal{A}=\left(\begin{array}{cc}
A+B & 0 \\
0 & A-B
\end{array}\right) .
$$

$\tilde{\Sigma}^{2}$ admits a manifestly positive-definite heat kernel regularization. Therefore, using Schwinger's proper time scheme, the real part of the effective action can be rewritten as

$$
W_{\mathbb{R}}=\frac{1}{8} \int_{0}^{\infty} \frac{d T}{T} \operatorname{Tr}_{16} e^{-\frac{\varepsilon}{2} T \tilde{\Sigma}^{2}},
$$

where $\mathcal{E}$ is the einbein, to be discussed further later.

This 16-dimensional representation of $\tilde{\Sigma}^{2}$ is useful because it is conveniently cast into a path integral in terms of Grassmanian variables. These variables are eigenvalues of coherent states of creation/annihilation operators that generate finite dimensional representations of the internal symmetries of the theory $[55,56]$. One obtains after some algebra,

$$
\begin{aligned}
W_{\mathbb{R}}= & \frac{1}{8} \int_{0}^{\infty} \frac{d T}{T} \mathcal{N}(T) \int_{P} \mathcal{D} x \int_{A P} \mathcal{D} \psi \mathcal{D} \lambda^{\dagger} \mathcal{D} \lambda \mathcal{J}\left(\lambda^{\dagger} \lambda\right) \\
& \times\left(e^{-\int_{0}^{T} d \tau \mathcal{L}_{L}(\tau)}+e^{-\int_{0}^{T} d \tau \mathcal{L}_{R}(\tau)}\right) .
\end{aligned}
$$

Some details of this derivation are given in [43]. The point particle Lagrangian for left/right chiralities is

$$
\begin{aligned}
\mathcal{L}_{L / R}(\tau)= & \frac{\dot{x}^{2}}{2 \mathcal{E}}+\frac{1}{2} \psi_{a} \dot{\psi}_{a}+\lambda^{\dagger} \dot{\lambda} \\
& -\lambda^{\dagger}\left[i \dot{x}_{\mu}(A \pm B)_{\mu}-\frac{i \mathcal{E}}{2} \psi_{\mu} \psi_{\nu} F_{\mu \nu}[A \pm B]\right] \lambda,
\end{aligned}
$$

with the normalization $\mathcal{N}(T)=\int \mathcal{D} p e^{-\frac{\varepsilon}{2} \int_{0}^{T} d \tau p^{2}(\tau)}$. Here $\psi_{a}=\sqrt{2}\left\langle\psi\left|\Gamma_{a}\right| \psi\right\rangle$, with $a=1, \ldots, 6$ are Grassmann variables, which are defined over a real vector space, where $|\psi\rangle$ represents a coherent state basis of the Clifford algebra. Likewise, $\lambda^{\dagger}$ and $\lambda$ are independent Grassmanian eigenvalues respectively of creation and annihilation fermion operators that generate finite dimensional representation of $S U\left(N_{c}\right)$, where $N_{c}$ is the number of colors. The factor $\mathcal{J}\left(\lambda^{\dagger} \lambda\right)=$ $\left(\frac{\pi}{T}\right)^{N_{c}} \sum_{\phi} \exp \left[i \phi\left(\lambda^{\dagger} \lambda+N_{c} / 2-1\right)\right]$ is required to project intermediate states in the path integral on to coherent states with unit occupancy [73]. The labels $P$ and $A P$ denote periodic and antiperiodic boundary conditions for the configuration space and Grassmanian variables respectively. Henceforth, the Abelian reduction of Eq. (11) will be sufficient for our purposes; the extension of our discussion to color degrees of freedom is straightforward [47-49].

Varying the real part of the effective action with respect to the vector gauge field gives the vector current,

$$
\begin{aligned}
\left\langle j_{\mu}^{V}(y)\right\rangle= & \frac{\delta \Gamma_{\mathbb{R}}}{\delta A_{\mu}(y)}=-\frac{i}{8} \int_{0}^{\infty} \frac{d T}{T} \mathcal{N} \int_{P} \mathcal{D} x \int_{A P} \mathcal{D} \psi j_{\mu}^{V, c l} \\
& \times\left(e^{-\int_{0}^{T} d \tau \mathcal{L}_{L}(\tau)}+e^{-\int_{0}^{T} d \tau \mathcal{L}_{R}(\tau)}\right),
\end{aligned}
$$

with $j_{\mu}^{V, c l}(y)=\int_{0}^{T} d \tau\left[\mathcal{E} \psi_{\nu} \psi_{\mu} \partial_{\nu}-\dot{x}_{\mu}\right] \delta^{4}(x(\tau)-y)$. This satisfies both $\partial_{\mu} j_{\mu}^{V, c l}=0$ and $\partial_{\mu}\left\langle j_{\mu}^{V}\right\rangle=0$.
The imaginary relative phase in the effective action can be written as

$$
W_{\llbracket}=-\frac{1}{2} \arg \operatorname{det}[\Omega], \quad \Omega=\left(\begin{array}{ll}
0 & \theta \\
\theta & 0
\end{array}\right),
$$

where $\theta$ is given in Eq. (2) and the matrix $\Omega$ is

$$
\Omega=\Gamma_{\mu}\left(p_{\mu}-A_{\mu}\right)-i \Gamma_{7} \Gamma_{\mu} \Gamma_{5} \Gamma_{6} B_{\mu} .
$$

The D'Hoker and Gagné [72,73] trick consists of introducing a parameter that regulates chiral symmetry breakingdistinct from those employed previously [71] - to write $W_{\square}$ as

$$
W_{\square}=\frac{i \mathcal{E}}{64} \int_{-1}^{1} d \alpha \int_{0}^{\infty} d T \operatorname{Tr}\left\{\hat{M} e^{-\frac{\mathcal{E}}{2} T \tilde{\Sigma}_{(\alpha)}^{2}}\right\},
$$

with a trace insertion

$\hat{M}=\Gamma_{7}\left(2 \Gamma_{5} \Gamma_{6}\left[\partial_{\mu}, B_{\mu}\right]+\left[\Gamma_{\mu}, \Gamma_{\nu}\right]\left\{\partial_{\mu}, B_{\nu}\right\} \Gamma_{5} \Gamma_{6}\right) \rrbracket_{2}$,

that is linear in the axial-vector field and diagonal in the two dimensional field representation space introduced in Eq. (9). $\tilde{\Sigma}_{(\alpha)}^{2}$ is identical to the expression in Eq. (6), with $B \rightarrow \alpha B$, where $\alpha$ breaks chiral symmetry explicitly for $\alpha \neq \pm 1$.

This form of the phase of the fermion determinant is useful because it has a heat-kernel structure that can be computed, in a manner identical to the real part, using Grassmanian path integrals [55,56]. The path integral representation of $W_{\square}$ is given in [43]; it is further shown explicitly there that this representation gives the wellknown anomaly relation

$$
\begin{aligned}
\partial_{\mu}\left\langle j_{\mu}^{5}(y)\right\rangle & \left.\equiv \partial_{\mu} \frac{i \delta W_{\square}}{\delta B_{\mu}(y)}\right|_{B=0} \\
& =-\frac{1}{16 \pi^{2}} \epsilon^{\mu \nu \rho \sigma} F_{\mu \nu}(y) F_{\rho \sigma}(y) .
\end{aligned}
$$

\section{BERRY'S PHASE FROM THE REAL PART OF THE QED EFFECTIVE ACTION}

From the discussion above, it is clear that the chiral anomaly arises from the imaginary part of the effective action $W_{\rrbracket}$. We will now show instead that Berry's phase arises from taking a nonrelativistic and adiabatic limit of the real part of the effective action $W_{\mathbb{R}}$. Our starting point is the worldline Lagrangian in Eq. (12) continued to Minkowskian metric $(g=\operatorname{diag}[-,+,+,+])$. We proceed by introducing Lagrange multipliers in Eq. (12) to (i) impose the mass-shell constraint and (ii) to project out unphysical spin degrees of freedom for both massless and massive point particles. After imposing all constraints, ${ }^{5}$

\footnotetext{
${ }^{5}$ The integration over the parameter $T$ represents the diffeomorphism invariance of the worldline action-it can either be performed explicitly $[74,75]$ or fixed equivalently for a choice of the einbein that respects the constrained Hamiltonian dynamics of massless and massive spinning particles. Since we are interested in real-time dynamics, we will adopt the latter approach.
} 
and eliminating thereby unphysical degrees of freedom, ${ }^{6}$ the Lagrangian can be written as $S=\int d \tau \mathcal{L}$ (setting $\tau=c t \sqrt{1-\mathbf{v}^{2} / c^{2}}$, with $x^{\mu}=(c t, \vec{x})$, defining $z=\sqrt{-\dot{x}^{2}}$ and putting the auxiliary field $B=0$ ),

$$
\begin{aligned}
\mathcal{L}= & -\frac{m_{R} c z}{2}\left(1+\frac{m^{2}}{m_{R}^{2}}\right)+\frac{i}{2}\left(\boldsymbol{\mu} \boldsymbol{\psi} \boldsymbol{\gamma}-\psi_{0} \dot{\psi}_{0}\right) \\
& +\frac{\dot{x}_{\mu} A^{\mu}(x)}{c}-\frac{i z}{m_{R} c} \psi^{0} F_{0 i} \psi^{i}-\frac{i z}{2 m_{R} c} \psi^{i} F_{i j} \psi^{j},
\end{aligned}
$$

where $m_{R}^{2}=m^{2}+i \psi^{\mu} F_{\mu \nu} \psi^{\nu} / c^{2}$ is the effective mass for the spinning worldline. The spin three-vector can be defined as $S^{i}=-\frac{i}{2} \epsilon^{i j k} \psi^{j} \psi^{k}$, where $\epsilon^{i j k}$ is the Levi-Civita symbol. Likewise, the magnetic field $B^{i}=$ $\frac{1}{2} \epsilon^{i j k} F^{j k}$ and the electric field $E^{i}=F^{0 i}$. The EulerLagrange equations of motion are [43]

$$
\begin{aligned}
-\frac{\bar{m}}{Y} \frac{d^{2} x^{\mu}}{d u^{2}}+\frac{i Y}{2 \bar{m}} \psi^{\alpha} F_{\alpha \beta} \psi^{\beta}+F^{\mu \nu} \frac{d x_{\nu}}{d u} & =0, \\
\frac{d \psi_{\mu}}{d u}-\frac{Y}{\bar{m}} F_{\mu \nu} \psi^{\nu} & =0,
\end{aligned}
$$

which represent the covariant form of the BargmannMichel-Telegdi [47-51,57] and Wong's equations [76]. We have abbreviated $u \equiv \tau / T, Y=\sqrt{-\int_{0}^{1} d u(d x / d u)^{2}}$ and $\bar{m}=\sqrt{m^{2}+i \int_{0}^{1} d u \psi^{\mu} F_{\mu \nu} \psi^{\nu}}$. The last two terms in Eq. (19) are respectively,

$$
-i \psi^{0} F_{0 i} \psi^{i}=\frac{\boldsymbol{S} \cdot(\boldsymbol{P} \times \boldsymbol{E})}{c P^{0}} ;-\frac{i}{2} \psi^{i} F_{i j} \psi^{j}=\boldsymbol{S} \cdot \boldsymbol{B} .
$$

Here $P^{\mu} \equiv p^{\mu}-A^{\mu}$, where $p^{\mu}$ is the canonical momentum. To take the nonrelativistic limit, we expand the effective mass as $m_{R} \approx m(1+X)$ with $X=-\left(\boldsymbol{S} \cdot(\boldsymbol{P} \times \boldsymbol{E}) /\left[c P^{0}\right]+\right.$ $\boldsymbol{S} \cdot \boldsymbol{B}) /\left(2 m^{2} c^{2}\right)$. Observing that $X \propto(v / c)^{2}$, where $\vec{v}$ is the three-velocity of the spinning worldline, one can expand Eq. (19) to obtain

$$
\begin{aligned}
\mathcal{L}_{\mathrm{NR}}= & -m c^{2}+\frac{1}{2} m \boldsymbol{v}^{2}+\frac{i}{2}\left(\boldsymbol{\psi} \boldsymbol{\dot { \psi }}-\psi_{0} \dot{\psi}_{0}\right)-A^{0}+\frac{\boldsymbol{v}}{c} \cdot \boldsymbol{A} \\
& +\frac{\boldsymbol{S} \cdot\left(\left[\boldsymbol{v} / c-\boldsymbol{A} /\left(m c^{2}\right)\right] \times \boldsymbol{E}\right)}{m c}+\frac{\boldsymbol{S} \cdot \boldsymbol{B}}{m}+O\left(\frac{v^{3}}{c^{3}}\right) .
\end{aligned}
$$

Compactly expressing the nonrelativistic action as $S=$ $\int d t\left(\boldsymbol{p} \cdot \dot{\boldsymbol{x}}+\frac{i}{2} \boldsymbol{\psi} \cdot \dot{\boldsymbol{\psi}}-H\right)$, the corresponding Hamiltonian is ${ }^{7}$

\footnotetext{
${ }^{6}$ A detailed discussion of the constrained Hamilton dynamics of worldlines can be found in [43].

The variable $\psi_{0}$ is not dynamical and we will drop it henceforth.
}

$$
\begin{aligned}
H \equiv & m c^{2}+\frac{\left(\boldsymbol{p}-\frac{\boldsymbol{A}}{c}\right)^{2}}{2 m}+A^{0}(x) \\
& -\frac{\boldsymbol{S} \cdot\left(\left[\boldsymbol{v} / c-\boldsymbol{A} /\left(m c^{2}\right)\right] \times \boldsymbol{E}\right)}{2 m c}-\frac{\boldsymbol{B} \cdot \boldsymbol{S}}{m} .
\end{aligned}
$$

Expressed in this form, the nonrelativistic point particle Hamiltonian is familiar [77]; the penultimate term is the spin-orbit interaction energy from Thomas precession, while the final term is the Larmor interaction energy. In atomic physics their combined effect is of course to reduce the Larmor energy by the famous "Thomas $1 / 2$ ".

In the following, we will show how the system described by Eq. (23) contains, in an adiabatic approximation, a Berry phase; in this limit, it has the monopole form postulated in [24-26,28]. To recover the expressions in [24,25] we requantize the spin, by promoting the spin (phase-space) variables $\psi$ to the Hilbert space operators $\psi_{i} \rightarrow \sqrt{\frac{\hbar}{2}} \sigma_{i} \equiv \hat{\psi}_{i}$ and $S_{i} \rightarrow \frac{\hbar}{2} \sigma_{i} \equiv \hat{S}_{k}$, where $\sigma$ are the Pauli matrices and hats indicate operators. Further, to describe the finite phase space of Grasmannian variables $\psi$, we define the two dimensional Hilbert space for a spin- $1 / 2$ particle at every point in phase space $(\boldsymbol{p}, \boldsymbol{x})$ by the eigenstates $\left|\psi^{ \pm}\right\rangle=$ $\left|\psi^{ \pm}(\boldsymbol{p})\right\rangle$. Defining $\boldsymbol{n}=\frac{\mathbf{p}}{|\mathbf{p}|} \equiv(\sin \theta \cos \phi, \sin \theta \sin \phi, \cos \theta)$, one has two choices

$$
\begin{gathered}
\left|\psi_{+}^{(1)}(\boldsymbol{p})\right\rangle=\frac{N}{2}(1+\boldsymbol{n} \cdot \boldsymbol{\sigma})\left(\begin{array}{l}
1 \\
0
\end{array}\right)=\left(\begin{array}{c}
\cos \frac{\theta}{2} \\
e^{i \phi} \sin \frac{\theta}{2}
\end{array}\right) \\
\left|\psi_{+}^{(2)}(\boldsymbol{p})\right\rangle=\frac{N}{2}(1+\boldsymbol{n} \cdot \boldsymbol{\sigma})\left(\begin{array}{l}
0 \\
1
\end{array}\right)=\left(\begin{array}{c}
e^{-i \phi} \cos \frac{\theta}{2} \\
\sin \frac{\theta}{2}
\end{array}\right),
\end{gathered}
$$

for the "spin up" + basis vectors (where $N$ is a normalization factor) and similarly for the "spin down" basis vectors. (See also [78].) The two choices of basis vectors are not defined globally for all $\boldsymbol{p}$ with Eq. (24) [Eq. (25)] ill defined for the south (north) pole for $\theta=\pi(0)$. One set can however be used for the northern hemisphere and the other for the southern one, and are related as $\left|\psi_{+}^{(1)}(\boldsymbol{p})\right\rangle=e^{i \phi}\left|\psi_{+}^{(2)}(\boldsymbol{p})\right\rangle$ [78].

These basis states allow us to derive a path integral formulation in the adiabatic limit of the theory defined by Eq. (23). The transition amplitude for the Hamiltonian operator corresponding to Eq. (23) from an initial state $\left|\psi^{+}\left(\mathbf{p}_{i}\right)\right\rangle$ at time $t_{i}$ to the state with momentum $\mathbf{p}_{f}$ at finite time $t_{f}$ is

$T\left(\mathbf{p}_{f}, \mathbf{p}_{i},+\right) \equiv\left\langle\mathbf{p}_{f}, \psi^{+}\left(\mathbf{p}_{f}\right)\left|e^{-i \hat{H}\left(t_{f}-t_{i}\right)}\right| \mathbf{p}_{i}, \psi^{+}\left(\mathbf{p}_{i}\right)\right\rangle$.

The construction of the path integral for this amplitude requires insertions of complete sets of intermediate states

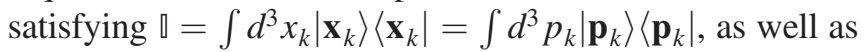
one for the two dimensional spin-Hilbert space: $\mathbb{\square}_{2}=\left|\psi^{+}\right\rangle\left\langle\psi^{+}|+| \psi^{-}\right\rangle\left\langle\psi^{-}\right|$. The adiabatic approximation corresponds to $\frac{B \cdot S}{2 m} \approx 0$. Therefore in this limit we can 
neglect the second term $\left|\psi^{-}\right\rangle\left\langle\psi^{-}\right|$, thereby constraining the dynamical spin degrees of freedom.

The transition matrix element can thus be written as

$$
\begin{aligned}
& T\left(\mathbf{p}_{f}, \mathbf{p}_{i},+\right) \\
& =\int\left(\prod_{k=1}^{N-1} d^{3} p_{k}\right)\left(\prod_{l=1}^{N} d^{3} x_{l}\right) \\
& \quad \times \prod_{j=1}^{N} \frac{1}{(2 \pi)^{3}} e^{-i \mathbf{x}_{j} \cdot\left(\mathbf{p}_{j}-\mathbf{p}_{j-1}\right)-i H_{j} \Delta}\left\langle\psi^{+}\left(\mathbf{p}_{j}\right) \mid \psi^{+}\left(\mathbf{p}_{j-1}\right)\right\rangle,
\end{aligned}
$$

where $\Delta \equiv\left(t_{f}-t_{i}\right) / N$ and $H_{j}$ is Eq. (23) evaluated at $\left(\boldsymbol{x}_{j}, \boldsymbol{p}_{j}\right)$. Taylor expanding $\left|\psi^{+}\left(\mathbf{p}_{j-1}\right)\right\rangle=\left\{1+\left[\mathbf{p}_{j}-\mathbf{p}_{j-1}\right]\right.$. $\left.\boldsymbol{\nabla}_{\boldsymbol{p}}\right\}\left|\psi^{+}\left(\mathbf{p}_{j}\right)\right\rangle+\cdots$, it is straightforward to show in the continuum limit that one obtains Berry's phase,

$$
\prod_{j=1}^{N}\left\langle\psi^{+}\left(\boldsymbol{p}_{j}\right) \mid \psi^{+}\left(\boldsymbol{p}_{j-1}\right)\right\rangle \rightarrow \exp \left(i \int d t \dot{\mathbf{p}} \cdot \mathcal{A}(\boldsymbol{p})\right),
$$

where $\mathcal{A}(\boldsymbol{p}) \equiv-i\left\langle\psi^{+}(\boldsymbol{p})\left|\boldsymbol{\nabla}_{p}\right| \psi^{+}(\boldsymbol{p})\right\rangle$ is the Berry connection. The final expression for the path integral is

$T\left(\mathbf{p}_{f}, \mathbf{p}_{i},+\right)=\int \mathcal{D} x \mathcal{D} p \exp \left(i \int d t[\dot{\mathbf{x}} \cdot \mathbf{p}-\tilde{H}]\right)$,

with $\tilde{H}=m c^{2}+\frac{(\boldsymbol{p}-\boldsymbol{A} / c)^{2}}{2 m}+A^{0}(x)-\dot{\mathbf{p}} \cdot \mathcal{A}(\boldsymbol{p})$. Eq. (29) is closely related to a similar formulation in [24-26,28].

We note a few crucial points in our derivation and interpretation for systems where the chemical potential is much smaller than the rest mass. First, as we showed, the structure of Berry's phase is restricted to the nonrelativistic adiabatic limit where the Larmor interaction energy is much smaller than the rest energy. It is ill-defined in the massless case albeit the spin basis vectors in Eq. (24) look similar to Weyl spinors. An exception holds for massless systems with a large chemical potential; the latter in that case takes over the role of the mass [43].

Our derivation further makes it clear that the topological structure of Berry's phase [79] is distinct from that of the chiral anomaly. The former arises from the real part of the QED/QCD effective action while the latter can be traced to its relative phase. This point is also stressed in Refs. [44-46] where it was argued that the Berry phase in Eq. (29) should not be confused with a Wess-Zumino term. In these papers, specific examples in condensed matter systems are worked out. In our work, the distinction between the two phenomena is demonstrated directly from the QED effective action.

\section{CHIRAL KINETIC THEORY}

In the previous section, we illustrated the appearance of a Berry phase in the adiabatic limit of QED and QCD. In contrast, the chiral anomaly is independent of any approximations and arises from the fundamental structure of the theory, embodied by fermionic zero modes in the worldline formulation. Since the CME dynamics in heavy-ion collisions is relativistic, and far from adiabatic, kinetic theory constructions that explicitly incorporate the Berry phase along the lines of Eq. (29) are insufficient for this case. ${ }^{8}$ Since the mass of the up and down quarks is much smaller than the temperature, we will consider here the formulation of a kinetic theory exclusively for chiral fermions. Finite mass will be treated separately in later work.

We will begin by expressing the many-body SchwingerKeldysh (SK) path integral in the language of worldlines as [58],

$$
Z=\int[d \xi] \exp (-G[\xi]) \int_{\mathcal{C}}[d A] \exp \left(i S_{\text {eff }}\right),
$$

where

$$
S_{\text {eff }}[A, \xi]=-\frac{1}{4} \int_{\mathcal{C}} d^{4} x F_{\mu \nu} F^{\mu \nu}+\Gamma[A, \xi] .
$$

Here $\xi \equiv \xi\left(x_{\text {init }}, x_{\text {init }}^{\prime}, \psi_{\text {init }}, \psi \psi_{\text {init }}^{\prime} ; A_{\text {init }}\right)$ represents the initial conditions on the Keldysh contour, which we will assume are distributed probabilistically as $\exp (-G[\xi])$, where $G$ is a positive-definite weight functional. Equation (30) and Eq. (31) were introduced in [58] for spinless colored particles; it was shown there that if one takes the saddle point of $\Gamma$, the dynamics of the matter fields is determined by a Liouville equation. For the relativistic fermions in our QED example, we will add extend phase space by adding spin degrees of freedom: $\left(x^{\mu}, p^{\mu}\right) \rightarrow\left(x^{\mu}, p^{\mu}, \psi^{\mu}\right)$. ${ }^{9}$ The equations of motion are derived from the chiral many-body Hamiltonian, ${ }^{10}$

$$
H=\frac{\varepsilon}{2}\left[P^{2}+i \psi^{\mu} F_{\mu \nu}(x) \psi^{\nu}\right]+\frac{i}{2} c_{+} \chi_{+}-\frac{i}{2} c_{-} \chi_{-},
$$

where

$$
c_{ \pm} \equiv \frac{1}{2}\left( \pm P_{\mu} \psi^{\mu}+\frac{i}{3} \epsilon^{\mu \nu \alpha \beta} P_{\mu} \psi_{\nu} \psi_{\alpha} \psi_{\beta}\right) .
$$

Here Eq. (32) is the constrained relativistic Hamiltonian corresponding to Eq. (12), whereby Eq. (33) enforces the Weyl-constraints and $\chi_{ \pm}$are anticommuting Lagrange multipliers [84]. ${ }^{11}$ The equations of motion corresponding to this Hamiltonian cannot be obtained by putting $m=0$ in Eq. (20), and instead are expressed as,

$$
\dot{x}^{\mu}=\varepsilon P^{\mu}+\frac{i}{2} \psi^{\mu} \bar{\chi}-\frac{1}{6} \epsilon^{\mu \nu \alpha \beta} \psi_{\nu} \psi_{\alpha} \psi_{\beta} \tilde{\chi},
$$

\footnotetext{
${ }^{8}$ This may also be the case for topological effects in the transport of chiral fermions in an astrophysical context, if the chemical potential is much smaller than the mass [80-83].

${ }^{9} \psi_{5}$ is not dynamical in the chiral limit.

${ }^{10}$ Because we are describing the classical phase space of $N$ particles, a sum over particle labels is always implied but omitted in our notation.

${ }^{11}$ Some relevant details are presented in Supplemental Material [85].
} 


$$
\begin{aligned}
\dot{\psi}^{\mu} & =\varepsilon F^{\mu \alpha} \psi_{\alpha}+\frac{P^{\mu}}{2} \bar{\chi}+\frac{i}{4} \epsilon^{\mu \nu \alpha \beta} P_{\beta} \psi_{\nu} \psi_{\alpha} \tilde{\chi}, \\
\dot{P}^{\mu}= & \varepsilon P_{\alpha} F^{\mu \alpha}-\frac{i \varepsilon}{2} \psi^{\alpha} \partial^{\mu} F_{\alpha \beta} \psi^{\beta}+\frac{i}{2} F^{\mu \alpha} \psi_{\alpha} \bar{\chi} \\
& -\frac{1}{12} \epsilon_{\alpha \beta \lambda \sigma} F^{\mu \alpha} \psi^{\beta} \psi^{\lambda} \psi^{\sigma} \tilde{\chi},
\end{aligned}
$$

with $\bar{\chi} \equiv\left(\chi_{+}+\chi_{-}\right) / 2$ and $\tilde{\chi} \equiv \chi_{+}-\chi_{-}$.

With these equations of motion in hand, Liouville's equation for chiral Dirac fermions reads as,

$$
\begin{aligned}
\{f, H\}= & f\left(\frac{\overleftarrow{\partial}}{\partial x^{\mu}}\left[\varepsilon P^{\mu}+\frac{i}{2} \psi^{\mu} \bar{\chi}-\frac{1}{6} \epsilon^{\mu \nu \alpha \beta} \psi_{\nu} \psi_{\alpha} \psi_{\beta} \tilde{\chi}\right]\right. \\
& +\frac{\overleftarrow{\partial}}{\partial P^{\mu}}\left[\varepsilon P_{\alpha} F^{\mu \alpha}-\frac{i \varepsilon}{2} \psi^{\alpha} \partial^{\mu} F_{\alpha \beta} \psi^{\beta}+\frac{i}{2} F^{\mu \alpha} \psi_{\alpha} \bar{\chi}\right. \\
& \left.-\frac{1}{12} \epsilon_{\alpha \beta \lambda \sigma} F^{\mu \alpha} \psi^{\beta} \psi^{\lambda} \psi^{\sigma} \tilde{\chi}\right]+\frac{\overleftarrow{\partial}}{\partial \psi^{\mu}}\left[\varepsilon F^{\mu \alpha} \psi_{\alpha}\right. \\
& \left.\left.+\frac{P^{\mu}}{2} \bar{\chi}+\frac{i}{4} \epsilon^{\mu \nu \alpha \beta} P_{\beta} \psi_{\nu} \psi_{\alpha} \tilde{\chi}\right]\right)=0
\end{aligned}
$$

where $f$ is the phase-space distribution function given by

$$
\begin{aligned}
f(x, p, \psi) \equiv & 4 i \epsilon \delta^{(4)}\left(x^{\mu}-x^{\mu}(\tau)\right) \delta^{(4)}\left(P^{\mu}-P^{\mu}(\tau)\right) \\
& \times \delta^{(4)}\left(\psi^{\mu}-\psi^{\mu}(\tau)\right) .
\end{aligned}
$$

The properties of this phase-space distribution are discussed further in Supplemental Material [85]. In Eq. (38), $x^{\mu}(\tau), P^{\mu}(\tau)$ and $\psi^{\mu}(\tau)$ are the solutions to Eqs. (34)-(36) in the background of $A$, with the initial conditions for their respective temporal evolution specified by the initial density matrix of the SK path integral in Eq. (30). Since these initial conditions are probabilistic, we can split $f(x, p, \psi)$ into average and fluctuating parts, $f \equiv \bar{f}+$ $\delta f$. Expressed in this way, $\langle f\rangle=\bar{f}$ coincides with the Boltzmann one-particle distribution function [58,86]. Expressed in terms of mean and fluctuating components, the ensemble-averaged Liouville equation reads

$$
\begin{aligned}
\bar{f} & \left(\frac{\overleftarrow{\partial}}{\partial x^{\mu}}\left[\varepsilon P^{\mu}+\frac{i}{2} \psi^{\mu} \bar{\chi}-\frac{\epsilon^{\mu \nu \alpha \beta}}{6} \psi_{\nu} \psi_{\alpha} \psi_{\beta} \tilde{\chi}\right]\right. \\
& \left.+\frac{\overleftarrow{\partial}}{\partial \psi^{\mu}}\left[\varepsilon \bar{F}^{\mu \alpha} \psi_{\alpha}+\frac{P^{\mu}}{2} \bar{\chi}+\frac{i}{4} \epsilon^{\mu \nu \alpha \beta} P_{\beta} \psi_{\nu} \psi_{\alpha} \tilde{\chi}\right]\right) \\
& +\frac{\overleftarrow{\partial}}{\partial P^{\mu}}\left[\varepsilon \bar{F}^{\mu \alpha} P_{\alpha}-\frac{i \varepsilon}{2} \psi^{\alpha} \partial^{\mu} \bar{F}_{\alpha \beta} \psi^{\beta}+\frac{i}{2} \bar{F}^{\mu \alpha} \psi_{\alpha} \bar{\chi}\right. \\
& \left.-\frac{\epsilon_{\alpha \beta \lambda \sigma}}{12} \bar{F}^{\mu \alpha} \psi^{\beta} \psi^{\lambda} \psi^{\sigma} \tilde{\chi}\right]=C[\delta f, \delta F],
\end{aligned}
$$

where the fluctuation induced collision term is given by

$$
\begin{aligned}
C[\delta f, \delta F] & \\
\equiv & -\varepsilon\left\langle\delta f \frac{\overleftarrow{\partial}}{\partial \psi^{\mu}} \delta F^{\mu \nu}\right\rangle \psi_{\nu}+\frac{i \varepsilon}{2}\left\langle\delta f \frac{\overleftarrow{\partial}}{\partial P^{\mu}} \partial^{\mu} \delta F_{\alpha \beta}\right\rangle \psi^{\alpha} \psi^{\beta} \\
& -\left\langle\delta f \frac{\overleftarrow{\partial}}{\partial P^{\mu}} \delta F^{\mu \alpha}\right\rangle\left(\varepsilon P_{\alpha}+\frac{i}{2} \psi_{\alpha} \bar{\chi}-\frac{1}{12} \epsilon_{\alpha \beta \lambda \sigma} \psi^{\beta} \psi^{\lambda} \psi^{\sigma} \tilde{\chi}\right) .
\end{aligned}
$$

A similar expression is found for $\delta f$ and equations for higher correlation functions can be derived. The result is an infinite tower of coupled equations, analogous to the BogoliubovBorn-Green-Kirkwood-Yvon hierarchy [87-89]. In a dilute limit, known as the second moment approximation, one can expand to linear order in the fluctuations,

$$
\begin{gathered}
\delta f\left(\frac{\overleftarrow{\partial}}{\partial x^{\mu}}\left[\varepsilon P^{\mu}+\frac{i}{2} \psi^{\mu} \bar{\chi}-\frac{1}{6} \epsilon^{\mu \nu \alpha \beta} \psi_{\nu} \psi_{\alpha} \psi_{\beta} \tilde{\chi}\right]+\frac{\overleftarrow{\partial}}{\partial P^{\mu}}\left[\varepsilon \bar{F}^{\mu \alpha} P_{\alpha}\right.\right. \\
\left.-\frac{i \varepsilon}{2} \psi^{\alpha} \partial^{\mu} \bar{F}_{\alpha \beta} \psi^{\beta}+\frac{i}{2} \bar{F}^{\mu \alpha} \psi_{\alpha} \bar{\chi}-\frac{1}{12} \epsilon_{\alpha \beta \lambda \sigma} \bar{F}^{\mu \alpha} \psi^{\beta} \psi^{\lambda} \psi^{\sigma} \tilde{\chi}\right] \\
+\frac{\grave{\partial}}{\partial \psi^{\mu}}\left[\varepsilon \bar{F}^{\mu \alpha} \psi_{\alpha}+\frac{P^{\mu}}{2} \bar{\chi}+\frac{i}{4} \epsilon^{\mu \nu \alpha \beta} P_{\beta} \psi_{\nu} \psi_{\alpha} \tilde{\chi}\right]=K[\delta F],
\end{gathered}
$$

where

$$
\begin{aligned}
K[\delta F] \equiv & -\bar{f}\left(\frac { \overleftarrow { \partial } } { \partial P ^ { \mu } } \left[\varepsilon \delta F^{\mu \alpha} P_{\alpha}-\frac{i \varepsilon}{2} \psi^{\alpha} \partial^{\mu} \delta F_{\alpha \beta} \psi^{\beta}\right.\right. \\
& \left.+\frac{i}{2} \delta F^{\mu \alpha} \psi_{\alpha} \bar{\chi}-\frac{\epsilon_{\alpha \beta \lambda \sigma}}{12} \delta F^{\mu \alpha} \psi^{\beta} \psi^{\lambda} \psi^{\sigma} \tilde{\chi}\right] \\
& \left.+\frac{\overleftarrow{\partial}}{\partial \psi^{\mu}}\left[\varepsilon \delta F^{\mu \alpha} \psi_{\alpha}\right]\right) .
\end{aligned}
$$

The equations for $\delta f$ and $\bar{f}$ are supplemented by Maxwell's (Yang-Mills) equations, analogously split into background and fluctuating parts. Note that while $\varepsilon$ and $\chi_{ \pm}$appear in the equations, physical observables should not depend on these gauge parameters. They can be fixed, with suitable choices, as discussed in Supplemental Material [85].

The resulting set of equations can be solved in closed form under systematic approximations for specific examples [89]. One nontrivial example is that of the real time formulation [59] of a pseudoclassical kinetic theory from the worldline action for spinless colored particles [58]. It was shown there that the analogous set of coupled equations is equivalent to the non-Abelian BoltzmannLangevin Bödeker kinetic theory of hot QCD [86,90]. A novel element here is the systematic treatment of the chiral anomaly and the role of the resulting real time sphaleron transitions in the spacetime evolution of the chiral magnetic current. The explicit derivation of this "anomalous Bödeker theory" [91,92], including as well the matching of chiral kinetic theory to classical-statistical simulations at early times [12-14], and anomalous hydrodynamics [19-22] at late times, will be presented in followup work [60]. 


\section{ACKNOWLEDGMENTS}

R. V. thanks the Institut für Theoretische Physik, Heidelberg for their kind hospitality and the Excellence Initiative of Heidelberg University for a Guest Professorship during the period when this work was initiated; we thank Juergen Berges, Jan Pawlowski, and Michael Schmidt for encouraging this effort. We thank Cristina Manuel and Naoki Yamamoto for valuable comments. We thank Fiorenzo Bastianelli for many helpful discussions and for sharing his deep insights into the worldline formulation of quantum field theory. R. V. would also like to thank the attendees of a seminar on this work at Stony Brook for their helpful comments; in particular, he would like to thank Dima Kharzeev, Ho-Ung Yee, Yi Yin and Ismail Zahed. N. M. acknowledges support by the Studienstiftung des Deutschen Volkes and by the DFG Collaborative Research Centre SFB 1225 (ISOQUANT). This material is partially based upon work supported by the U.S. Department of Energy, Office of Science, Office of Nuclear Physics, under Contract No. DE- SC0012704, and within the framework of the Beam Energy Scan Theory (BEST) Topical Collaboration.
[1] A. Riotto and M. Trodden, Recent progress in baryogenesis, Annu. Rev. Nucl. Part. Sci. 49, 35 (1999).

[2] A. G. Cohen, D. B. Kaplan, and A. E. Nelson, Progress in electroweak baryogenesis, Annu. Rev. Nucl. Part. Sci. 43, 27 (1993).

[3] D. E. Kharzeev, L. D. McLerran, and H. J. Warringa, The Effects of topological charge change in heavy ion collisions: "Event by event P and CP violation", Nucl. Phys. A803, 227 (2008).

[4] K. Fukushima, D. E. Kharzeev, and H. J. Warringa, The chiral magnetic effect, Phys. Rev. D 78, 074033 (2008).

[5] D. E. Kharzeev, J. Liao, S. A. Voloshin, and G. Wang, Chiral magnetic and vortical effects in high-energy nuclear collisions-A status report, Prog. Part. Nucl. Phys. 88, 1 (2016).

[6] V. Skokov, P. Sorensen, V. Koch, S. Schlichting, J. Thomas, S. Voloshin, G. Wang, and H. U. Yee, Chiral magnetic effect task force report, Chin. Phys. C 41, 072001 (2017).

[7] Q. Li, D. E. Kharzeev, C. Zhang, Y. Huang, I. Pletikosić, A. V. Fedorov, R. D. Zhong, J. A. Schneeloch, G. D. Gu, and T. Valla, Observation of the chiral magnetic effect in ZrTe5, Nat. Phys. 12, 550 (2016).

[8] V. Skokov, A. Y. Illarionov, and V. Toneev, Estimate of the magnetic field strength in heavy-ion collisions, Int. J. Mod. Phys. A 24, 5925 (2009).

[9] W. T. Deng and X. G. Huang, Event-by-event generation of electromagnetic fields in heavy-ion collisions, Phys. Rev. C 85, 044907 (2012).

[10] M. Mace, S. Schlichting, and R. Venugopalan, Offequilibrium sphaleron transitions in the Glasma, Phys. Rev. D 93, 074036 (2016).

[11] G. D. Moore and M. Tassler, The sphaleron rate in SU(N) gauge theory, J. High Energy Phys. 02 (2011) 105.

[12] N. Tanji, N. Mueller, and J. Berges, Transient anomalous charge production in strong-field QCD, Phys. Rev. D 93, 074507 (2016).

[13] N. Müller, S. Schlichting, and S. Sharma, Chiral Magnetic Effect and Anomalous Transport from Real-Time Lattice Simulations, Phys. Rev. Lett. 117, 142301 (2016).

[14] M. Mace, N. Mueller, S. Schlichting, and S. Sharma, Nonequilibrium study of the chiral magnetic effect from real-time simulations with dynamical fermions, Phys. Rev. D 95, 036023 (2017).

[15] D. T. Son and A. R. Zhitnitsky, Quantum anomalies in dense matter, Phys. Rev. D 70, 074018 (2004).

[16] D. E. Kharzeev and H. U. Yee, Chiral magnetic wave, Phys. Rev. D 83, 085007 (2011).

[17] J. Berges, K. Boguslavski, S. Schlichting, and R. Venugopalan, Turbulent thermalization process in heavy-ion collisions at ultrarelativistic energies, Phys. Rev. D 89, 074011 (2014).

[18] A. Kurkela and Y. Zhu, Isotropization and Hydrodynamization in Weakly Coupled Heavy-Ion Collisions, Phys. Rev. Lett. 115, 182301 (2015).

[19] V. P. Nair, R. Ray, and S. Roy, Fluids, anomalies and the chiral magnetic effect: A group-theoretic formulation, Phys. Rev. D 86, 025012 (2012).

[20] M. Hongo, Y. Hirono, and T. Hirano, First numerical simulations of anomalous hydrodynamics, Phys. Lett. B 775, 266 (2017).

[21] D. Karabali and V. P. Nair, Relativistic particle and relativistic fluids: Magnetic moment and spin-orbit interactions, Phys. Rev. D 90, 105018 (2014).

[22] Y. Hirono, T. Hirano, and D. E. Kharzeev, The chiral magnetic effect in heavy-ion collisions from event-by-event anomalous hydrodynamics, arXiv:1412.0311.

[23] M. V. Berry, Quantal phase factors accompanying adiabatic changes, Proc. R. Soc. A 392, 45 (1984).

[24] D. T. Son and N. Yamamoto, Berry Curvature, Triangle Anomalies, and the Chiral Magnetic Effect in Fermi Liquids, Phys. Rev. Lett. 109, 181602 (2012).

[25] M. A. Stephanov and Y. Yin, Chiral Kinetic Theory, Phys. Rev. Lett. 109, 162001 (2012).

[26] D. T. Son and N. Yamamoto, Kinetic theory with Berry curvature from quantum field theories, Phys. Rev. D 87, 085016 (2013).

[27] J. W. Chen, S. Pu, Q. Wang, and X. N. Wang, Berry Curvature and Four-Dimensional Monopoles in the Relativistic Chiral Kinetic Equation, Phys. Rev. Lett. 110, 262301 (2013).

[28] J. W. Chen, J. y. Pang, S. Pu, and Q. Wang, Kinetic equations for massive Dirac fermions in electromagnetic field with non-Abelian Berry phase, Phys. Rev. D 89, 094003 (2014). 
[29] J. Y. Chen, D. T. Son, M. A. Stephanov, H. U. Yee, and Y. Yin, Lorentz Invariance in Chiral Kinetic Theory, Phys. Rev. Lett. 113, 182302 (2014).

[30] G. Basar, D. E. Kharzeev, and I. Zahed, Chiral and Gravitational Anomalies on Fermi Surfaces, Phys. Rev. Lett. 111, 161601 (2013).

[31] M. Stone and V. Dwivedi, Classical version of the nonAbelian gauge anomaly, Phys. Rev. D 88, 045012 (2013).

[32] V. Dwivedi and M. Stone, Classical chiral kinetic theory and anomalies in even space-time dimensions, J. Phys. A 47, 025401 (2013).

[33] M. Stone, V. Dwivedi, and T. Zhou, Berry phase, Lorentz covariance, and anomalous velocity for Dirac and Weyl particles, Phys. Rev. D 91, 025004 (2015).

[34] C. Manuel and J.M. Torres-Rincon, Chiral transport equation from the quantum Dirac Hamiltonian and the on-shell effective field theory, Phys. Rev. D 90, 076007 (2014).

[35] C. Manuel and J. M. Torres-Rincon, Dynamical evolution of the chiral magnetic effect: Applications to the quark-gluon plasma, Phys. Rev. D 92, 074018 (2015).

[36] N. Yamamoto, Chiral transport of neutrinos in supernovae: Neutrino-induced fluid helicity and helical plasma instability, Phys. Rev. D 93, 065017 (2016).

[37] Y. Sun, C. M. Ko, and F. Li, Anomalous transport model study of chiral magnetic effects in heavy ion collisions, Phys. Rev. C 94, 045204 (2016).

[38] Y. Hidaka, S. Pu, and D. L. Yang, Relativistic chiral kinetic theory from quantum field theories, Phys. Rev. D 95, 091901 (2017).

[39] N. Yamamoto, Photonic chiral vortical effect, Phys. Rev. D 96, 051902 (2017).

[40] J.h. Gao, S. Pu, and Q. Wang, Covariant chiral kinetic equation in the Wigner function approach, Phys. Rev. D 96, 016002 (2017).

[41] S. Pu and A. Yamamoto, Abelian and non-Abelian Berry curvatures in lattice QCD, arXiv:1712.02218.

[42] A. Huang, S. Shi, Y. Jiang, J. Liao, and P. Zhuang, Complete and consistent chiral transport from Wigner function formalism, arXiv:1801.03640.

[43] N. Mueller and R. Venugopalan, Worldline construction of a covariant chiral kinetic theory, Phys. Rev. D 96, 016023 (2017).

[44] S. Deguchi and K. Fujikawa, Second quantized formulation of geometric phases, Phys. Rev. A 72, 012111 (2005).

[45] K. Fujikawa, Geometric phases and hidden local gauge symmetry, Phys. Rev. D 72, 025009 (2005).

[46] K. Fujikawa, Quantum anomaly and geometric phase: Their basic differences, Phys. Rev. D 73, 025017 (2006).

[47] A. P. Balachandran, P. Salomonson, B. S. Skagerstam, and J. O. Winnberg, Classical description of particle interacting with nonabelian gauge field, Phys. Rev. D 15, 2308 (1977).

[48] A. P. Balachandran, S. Borchardt, and A. Stern, Lagrangian and Hamiltonian descriptions of Yang-Mills particles, Phys. Rev. D 17, 3247 (1978).

[49] A. Barducci, R. Casalbuoni, and L. Lusanna, Classical scalar and spinning particles interacting with external YangMills fields, Nucl. Phys. B124, 93 (1977).
[50] A. Barducci, Pseudoclassical description of relativistic spinning particles with anomalous magnetic moment, Phys. Lett. 118B, 112 (1982).

[51] L. Brink, P. Di Vecchia, and P. S. Howe, A Lagrangian formulation of the classical and quantum dynamics of spinning particles, Nucl. Phys. B118, 76 (1977).

[52] D. M. Gitman, Path integrals and pseudoclassical description for spinning particles in arbitrary dimensions, Nucl. Phys. B488, 490 (1997).

[53] S. P. Gavrilov and D. M. Gitman, Quantization of spinning particle in arbitrary background, Classical Quantum Gravity 18, 2989 (2001).

[54] S. Naka, Canonical approach to spinning particles, Prog. Theor. Phys. 62, 278 (1979).

[55] F. A. Berezin and M. S. Marinov, Particle spin dynamics as the Grassmann variant of classical mechanics, Ann. Phys. (N.Y.) 104, 336 (1977).

[56] Y. Ohnuki and T. Kashiwa, Coherent states of Fermi operators and the path integral, Prog. Theor. Phys. 60, 548 (1978).

[57] V. Bargmann, L. Michel, and V. L. Telegdi, Precession of the Polarization of Particles Moving in a Homogeneous Electromagnetic Field, Phys. Rev. Lett. 2, 435 (1959).

[58] J. Jalilian-Marian, S. Jeon, R. Venugopalan, and J. Wirstam, Minding one's P's and Q's: From the one loop effective action in quantum field theory to classical transport theory, Phys. Rev. D 62, 045020 (2000).

[59] S. D. Mathur, Is the Polyakov path integral prescription too restrictive?, arXiv:hep-th/9306090.

[60] N. Mueller, R. Venugopalan, and Y. Yin (to be published).

[61] M. J. Strassler, Field theory without Feynman diagrams: One loop effective actions, Nucl. Phys. B385, 145 (1992).

[62] M. Mondragon, L. Nellen, M. G. Schmidt, and C. Schubert, Yukawa couplings for the spinning particle and the worldline formalism, Phys. Lett. B 351, 200 (1995).

[63] M. Mondragon, L. Nellen, M. G. Schmidt, and C. Schubert, Axial couplings on the worldline, Phys. Lett. B 366, 212 (1996).

[64] A. Hernandez, T. Konstandin, and M. G. Schmidt, Sizable $C P$ violation in the bosonized standard model, Nucl. Phys. B812, 290 (2009).

[65] C. Schubert, Perturbative quantum field theory in the string inspired formalism, Phys. Rep. 355, 73 (2001).

[66] F. Bastianelli and P. van Nieuwenhuizen, Path Integrals and Anomalies in Curved Space (Cambridge University Press, Cambridge, England, 2006).

[67] O. Corradini and C. Schubert, Spinning particles in quantum mechanics and quantum field theory, arXiv:1512.08694.

[68] K. Fujikawa, Path Integral Measure for Gauge Invariant Fermion Theories, Phys. Rev. Lett. 42, 1195 (1979).

[69] L. Alvarez-Gaume and E. Witten, Gravitational anomalies, Nucl. Phys. B234, 269 (1984).

[70] L. Alvarez-Gaume, Supersymmetry and the Atiyah-Singer index theorem, Commun. Math. Phys. 90, 161 (1983).

[71] A. M. Polyakov, Gauge fields and strings, Contemp. Concepts Phys. 3, 1 (1987).

[72] E. D'Hoker and D. G. Gagne, Worldline path integrals for fermions with scalar, pseudoscalar and vector couplings, Nucl. Phys. B467, 272 (1996). 
[73] E. D'Hoker and D. G. Gagne, Worldline path integrals for fermions with general couplings, Nucl. Phys. B467, 297 (1996).

[74] G. V. Dunne and C. Schubert, Worldline instantons and pair production in inhomogeneous fields, Phys. Rev. D 72, 105004 (2005).

[75] G. V. Dunne, Q.h. Wang, H. Gies, and C. Schubert, Worldline instantons. II. The fluctuation prefactor, Phys. Rev. D 73, 065028 (2006).

[76] S. K. Wong, Field and particle equations for the classical Yang-Mills field and particles with isotopic spin, Nuovo Cimento A 65, 689 (1970).

[77] J. J. Sakurai, Advanced Quantum Mechanics (AddisonWesley Publishers, Reading, MA, 1967).

[78] M. Stone, The Born-Oppenheimer approximation and the origin of Wess-Zumino terms: Some quantum mechanical examples, Phys. Rev. D 33, 1191 (1986); M. Stone and P. Goldbart, Mathematics for Physics II (Pimander-Casaubon, 2000).

[79] B. Simon, Holonomy, the Quantum Adiabatic Theorem, and Berry's Phase, Phys. Rev. Lett. 51, 2167 (1983).

[80] J. Charbonneau and A. Zhitnitsky, Topological currents in neutron stars: Kicks, precession, toroidal fields, and magnetic helicity, J. Cosmol. Astropart. Phys. 08 (2010) 010 .

[81] Y. Akamatsu and N. Yamamoto, Chiral Plasma Instabilities, Phys. Rev. Lett. 111, 052002 (2013).

[82] M. Dvornikov and V. B. Semikoz, Non-conservation of the neutrino current in a hot plasma of the early universe, Zh. Eksp. Teor. Fiz. 151, 860 (2017) [J. Exp. Theor. Phys. 124, 731 (2017)].
[83] D. B. Kaplan, S. Reddy, and S. Sen, Energy conservation and the chiral magnetic effect, Phys. Rev. D 96, 016008 (2017).

[84] A. Barducci, R. Casalbuoni, D. Dominici, and L. Lusanna, Pseudoclassical description of Weyl particles, Phys. Lett. 100B, 126 (1981).

[85] See Supplemental Material at http://link.aps.org/ supplemental/10.1103/PhysRevD.97.051901 for some relevant details of the many-body phase for Weyl particles in the world-line formulation. We discuss the properties of phase-space distributions for chiral particles and details of the Grassmann-extended canonical coordinates.

[86] D. F. Litim and C. Manuel, Effective transport equations for non-Abelian plasmas, Nucl. Phys. B562, 237 (1999).

[87] N. N. Bogoliubov, Kinetic equations, JETP 16, 691702 (1946); J. Yvon, La théorie statistique des uides et l'é quation d'é tat (in French), Actual. Sci. \& Indust. 203 (1937); J. G. Kirkwood, The statistical mechanical theory of transport processes I. general theory, J. Chem. Phys. 14, 180 (1946).

[88] S. de Groot et al., Relativistic Kinetic Theory (1980).

[89] L. D. Landau and E. M. Lifschits, Quantum Electrodynamics.

[90] D. Bödeker, From hard thermal loops to Langevin dynamics, Nucl. Phys. B559, 502 (1999).

[91] Y. Akamatsu and N. Yamamoto, Chiral Langevin theory for non-Abelian plasmas, Phys. Rev. D 90, 125031 (2014).

[92] Y. Akamatsu, A. Rothkopf, and N. Yamamoto, Non-Abelian chiral instabilities at high temperature on the lattice, J. High Energy Phys. 03 (2016) 210. 